\title{
Serum albumin to globulin ratio is related to cognitive decline via reflection of homeostasis: a nested case-control study
}

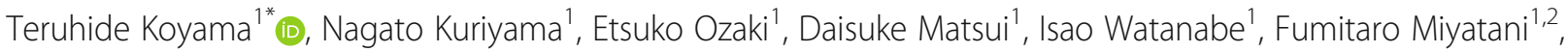
Masaki Kondo ${ }^{3}$, Aiko Tamura ${ }^{3}$, Takashi Kasai ${ }^{3}$, Yoichi Ohshima ${ }^{4}$, Tomokatsu Yoshida ${ }^{3}$, Takahiko Tokuda ${ }^{3,5}$, Ikuko Mizuta ${ }^{3}$, Shigeto Mizuno ${ }^{6}$, Kei Yamada ${ }^{7}$, Kazuo Takeda ${ }^{8}$, Sanae Matsumoto ${ }^{8}$, Masanori Nakagawa9

Toshiki Mizuno $^{3}$ and Yoshiyuki Watanabe ${ }^{1}$

\begin{abstract}
Background: Recent research suggests that several pathogenetic factors, including aging, genetics, inflammation, dyslipidemia, diabetes, and infectious diseases, influence cognitive decline (CD) risk. However, no definitive candidate causes have been identified. The present study evaluated whether certain serum parameters predict CD.
\end{abstract}

Methods: A total of 151 participants were assessed for CD using the Mini-Mental State Examination (MMSE), and 34 participants were identified as showing CD.

Results: Among CD predictive risk factors, Helicobacter pylori seropositivity was significantly predictive of CD risk, more so than classical risk factors, including white matter lesions and arterial stiffness [adjusted odds ratio $(O R)=4.786,95 \%$ confidence interval $(C I)=1.710-13.39]$. A multivariate analysis indicated that the albumin to globulin (A/G) ratio was the only factor that significantly lowered $C D$ risk $(\mathrm{OR}=0.092,95 \% \mathrm{Cl}=0.010-0.887)$. A/G ratio also was positively correlated with MMSE scores and negatively correlated with disruption of homeostatic factors (i.e., non-high-density lipoprotein, hemoglobin A1c, and high-sensitive C-reactive protein).

Conclusions: The current study results suggest that the $A / G$ ratio is related to cognitive decline and may reflect homeostatic alterations.

Keywords: Albumin to globulin ratio, Cognitive decline, Helicobacter pylori, Homeostatic alteration, Mini-mental state examination

\section{Background}

Improvements in health care support have greatly extended average life expectancy, resulting in a substantial increase in the number of elderly individuals worldwide. Some forms of memory impairment are observed among elderly adults and can be predictive of age-related cognitive decline associated with Alzheimer's disease (AD) [1] and other dementias. Rate of memory impairment varies based on several factors, including age, sex, types of cognitive tasks assessed, education, and emotional state [2].

\footnotetext{
* Correspondence: tkoyama@koto.kpu-m.ac.jp

${ }^{1}$ Department of Epidemiology for Community Health and Medicine, Kyoto Prefectural University of Medicine, 465 Kajii-cho, Kamigyo-ku, Kyoto 602-8566, Japan

Full list of author information is available at the end of the article
}

Previous reports have noted several causes for cognitive decline (CD). For instance, infection can cause both direct and indirect decrements. The association between Helicobacter pylori (H. pylori) infection and AD has recently been addressed [3], and other infections [i.e., Chlamydia pneumoniae (C. pneumoniae), cytomegalovirus, and herpes simplex virus type1] may influence AD manifestation [4]. Furthermore, inflammation-mediated damage in the apolipoprotein $\mathrm{E}(\mathrm{ApoE})$ allele 4 suggests a plausible marker for cognitive impairment, possibly due to increased viral replication, which could eventually lead to AD [5]. One way to affect this relationship is by controlling risk factors (e.g., diabetes, cholesterol, hypertension, stroke, or smoking) that could help alleviate physiological dementia risk factors [6]. A common factor is chronic and systemic 
inflammation, which leads to increased levels of several proinflammatory cytokines that subsequently promote CD progression [7]. Chronic and systemic inflammation also induces atherosclerosis [8] and atherosclerosis-promoted cognitive impairment [9].

There is growing interest in identifying individuals who have not yet demonstrated $C D$ but could be at greater risk for developing dementia. This is because cognitive impairment responds much better to treatment during early compared to advanced illness stages. With substantial increases in dementia incidence, early detection of possible precursors, diagnostics, treatment, and control of modifiable risk factors are highly important [10]. Insight is needed regarding the specific risk factors that predict $\mathrm{CD}$ incidence. Elucidation of these factors will help identify individuals with $C D$ who are at the highest risk for developing $\mathrm{AD}$ in the near future.

Thus, the aim of the present nested case control study was to evaluate whether certain serum parameters, commonly measured during routine health checkups including magnetic resonance imaging (MRI) and pulse wave velocity as a marker of arterial stiffness, could be viable predictors of $\mathrm{CD}$ incidence.

\section{Methods}

\section{Study participants}

The present study consisted of self-administered questionnaires and medical examinations, including blood tests, conducted at the Kyoto Industrial Health Association. From 2003 to 2004, 488 Japanese participants completed a baseline epidemiological survey [11]. Basic cognitive functioning was assessed for 273 participants from 2006 to 2008 and for 290 participants from 2012 to 2014. A group of 151 participants (101 men and 50 women), with normal cognition in 2006-2008, attended follow-up visits during both the 2006-2008 and 20122014 periods. We included all of these 151 patients in our study in order to avoid selection bias. The Ethics Board from the Kyoto Prefectural University of Medicine approved the study protocol (G-144). After we explained the purpose of the study, written informed consent was obtained from all participants.

\section{Cognitive testing}

The Mini-Mental State Examination (MMSE) is a brief, but universal, 30-point measure of cognitive status [12]. The MMSE has become one of the most widely used cognitive screening instruments for $\mathrm{CD}$, which covers various cognitive domains. Specifically, the MMSE is used to estimate the severity of cognitive impairment and assess longitudinal changes in cognitive status. Trained neurologists or a neuropsychologist determined the MMSE scores as described previously [13]. A score $\leq 27$ is considered reflective of cognitive impairment [14]. We were able to identify 34 participants as suitable for the CD group as they produced MMSE scores between 28-30 points in 2006-2008 and scores from 24-27 in 2012-2014. Similarly, 117 participants were defined as the control group, with scores from 28-30 in 2006-2008 that did not decrease when assessed in 2012-2014. The time between the two cognitive evaluations was not significantly different between the control (mean $=5.74$ years) and $\mathrm{CD}$ (mean $=5.76$ years) groups.

The verbal fluency test is a well-established method for evaluation of cognitive function [15]. All participants also completed a verbal fluency test. In this task, as in previous reports, the participants were asked to provide as many words beginning with Ta and Ka as they could recall [13].

\section{Medical information and blood biochemistry}

The present study evaluated medical information obtained via self-administered questionnaires (education level, anamnesis at baseline and in 2012-2014, medication, frequency of depressive symptoms, smoking, and drinking habits). Instrumental activities of daily living (IADL) and metabolic equivalents (METs) were assessed as previously reported [16, 17]. The scoring guidelines recommend adding an additional point for people with less than 13 years of education [18]. Furthermore, blood chemistry data [triglycerides, total cholesterol, high-density lipoprotein cholesterol (HDL-C), non-HDL-C, total protein, albumin, $\mathrm{A} / \mathrm{G}$ ratio, creatinine, uric acid, hemoglobin Alc (HbA1c), high sensitive C-reactive protein (hsCRP), and antibodies against $C$. pneumonia, and $H$. pylori antibodies] were assessed. An IgG index above 1.1, and an IgA index above 1.1, was defined as criteria for C. pneumoniae positivity [19], and a cutoff point greater than 2.3 for the ELISA VALUE indicated $H$. pylori positivity [20]. The following anthropometry data were also obtained during the health check-ups: weight, height, and systolic and diastolic blood pressure. Anamnesis and medication history were assessed using a questionnaire. Hypertension was resting systolic blood pressure $\geq 140 \mathrm{mmHg}$ or being treated for hypertension. Diabetes mellitus was defined as $\mathrm{HbA} 1 \mathrm{c} \geq 6.5 \%$ and dyslipidemia as triglycerides $\geq 150$ or HDL $\leq 40$. Additionally, the pulse wave velocity [21], which is a potential marker of arterial stiffness, was measured in 2006-2008 and 2012-2014.

\section{Apolipoprotein E genotyping}

Genomic DNA was extracted from the buffy coat fraction of each participant's blood sample. Genotyping was performed using polymerase chain reaction (PCR) with the following primers; forward: ACGAGACCATGAAG GAGTTGAA and reverse: ACCTGCTCCTTCACCTCG TCCAG. Amplification of the genomic DNA resulted in a $\mathrm{PCR}$ product $=514 \mathrm{bp}$, which was subjected to a direct 
sequence or PCR-restriction fragment length polymorphism analysis [22]. The ApoE isoforms differed in cysteine and arginine content at positions 112 and 158: ApoE- $\varepsilon 2:$ Cys (TGC), Cys (TGC), ApoE- $\varepsilon 3$ : Cys (TGC), Arg (CGC), ApoE- $\varepsilon 4$ : Arg (CGC), Arg (CGC). ApoE status was classified as $\varepsilon 4$ carriers for participants with the ApoE4 isoform (phenotypes $\varepsilon 2 / 4, \varepsilon 3 / 4, \varepsilon 4 / 4$ ) and as non-4 carriers for participants without the ApoE4 isoform (phenotypes $\varepsilon 2 / 2$, $\varepsilon 2 / 3, \varepsilon 3 / 3$ ).

\section{Scoring white matter and periventricular hyperintensities}

Brain MRI was performed using a 1.5-T scanner. MRI was performed to assess different types of hyperintense signal abnormalities surrounding the ventricles, and deep white matter abnormalities were evaluated as deep white matter lesions (DWL) and periventricular hyperintensities (PVH), as previously reported [13]. MRI cerebrovascular staging was carried out using the Fazekas classification [23].

\section{Statistical analyses}

Continuous variables are expressed as means \pm standard deviations (SDs) or median [range], and categorical data are expressed as sums and percentages. Inter-group comparisons were performed using unpaired $t$-tests for continuous variables or Mann-Whitney U-tests, and the chi-square or Fisher's exact tests for categorical variables (sex, ApoE4, education, depressive symptoms, baseline and 2012-2014 period anamnesis, C. pneumonia and $H$. pylori seropositivity, drinking and smoking prevalence, DWL, and PVH). Odds ratios (OR) and 95\% confidence intervals $(\mathrm{CI})$ were calculated using logistic regression analyses in which $\mathrm{CD}$ was the dependent variable and age, sex, ApoE4 status, education, smoking and drinking habits, and baseline anamnesis were the independent variables. Significant predictors from the logistic regression analysis were considered independent variables in the multiple logistic regression analysis using a stepwise forward selection method. A Spearman's rank correlation coefficient was calculated to confirm whether serum A/G ratio was related to MMSE scores, pulse wave velocity, and hsCRP, as well as significant variables from the logistic regression analysis. All statistical tests were two-tailed, and differences with a $p$-value $<0.05$ were considered statistically significant. SPSS software (version 18.0) was used for all statistical analyses.

\section{Results}

\section{Participant characteristics}

Table 1 shows participant characteristics, including anthropometric measures, blood chemistry data, questionnaire responses, and the number for each item between the control and CD groups. The mean age $( \pm$ standard deviation: SD) for the control group was 59.4 $( \pm 5.9)$ years, compared to $61.2( \pm 4.6)$ years for the CD group. There were no significant differences between the anthropometric measures of the two groups. Although the $\mathrm{CD}$ group did not show significantly decreased scores on verbal fluency tasks in 2006-2008, their verbal fluency scores significantly decreased in 2012-2014. Furthermore, no significant differences in depressive symptoms, IADL, or METs were observed between the control and CD groups. The distribution of ApoE4 genotypes was in the Hardy-Weinberg equilibrium (control group: $p=0.621$; CD group: $p=0.565$ ). The ApoE4 allele distribution was not significantly different between the control and CD groups.

\section{Associations between CD and control participant characteristics}

Non-HDL-C, total protein, HbA1c, H. pylori seropositivity, and pulse wave velocity during both 2006-2008 and 2012-2014 were significantly higher in the CD group compared to the control group (Table 1). In contrast, the $A / G$ ratio was significantly lower in the $C D$ group (Table 1).

To determine variables significantly associated with $\mathrm{CD}$, a logistic regression analysis adjusted for age, sex, ApoE4 status, education, smoking and alcohol drinking habits, and anamnesis was performed. The variables selected by this analysis were MRI evaluation, body mass index (BMI), systolic blood pressure (SBP), diastolic blood pressure (DBP), triglycerides, total cholesterol, HDL, non-HDL, total protein, albumin, A/G ratio, creatinine, uric acid, HbA1c, hsCRP, C. pneumoniae and H. pylori seropositivity, pulse wave velocity, education, and ApoE4 status (Tables 2 and 3). From a diagnostic imaging viewpoint (Table 2), the odds of DWL grade 1 and 2, which were evaluated by a Fazekas classification during the 2nd follow-up, showed significant higher values for $\mathrm{CD}$ group. As shown in Table 3, non-HDL-C, A/G ratio, HbAlc, and $H$. pylori seropositivity were predictive of $\mathrm{CD}$.

Next, a multivariate analysis was performed with all of the significant variables considered simultaneously: nonHDL-C, A/G ratio, HbA1c, and $H$. pylori seropositivity (Table 4). Based on a stepwise forward selection method, A/G ratio was significantly predictive with a low OR $(\mathrm{OR}=0.092,95 \% \mathrm{CI}=0.010-0.887)$, and $H$. pylori seropositivity was significantly predictive with a high $\mathrm{OR}$ $(\mathrm{OR}=4.468,95 \% \mathrm{CI}=1.535-13.00)$. Therefore, $\mathrm{A} / \mathrm{G}$ ratios were significantly positive correlation of MMSE scores (during both 2006-2008 and 2012-2014), and negative correlation with non-HDL-C, HbA1c, and hsCRP (Table 5).

\section{Discussion}

Although no single cause for cognitive impairment has been identified, recent research suggests that several pathogenetic factors such as aging, genetics, inflammation, 


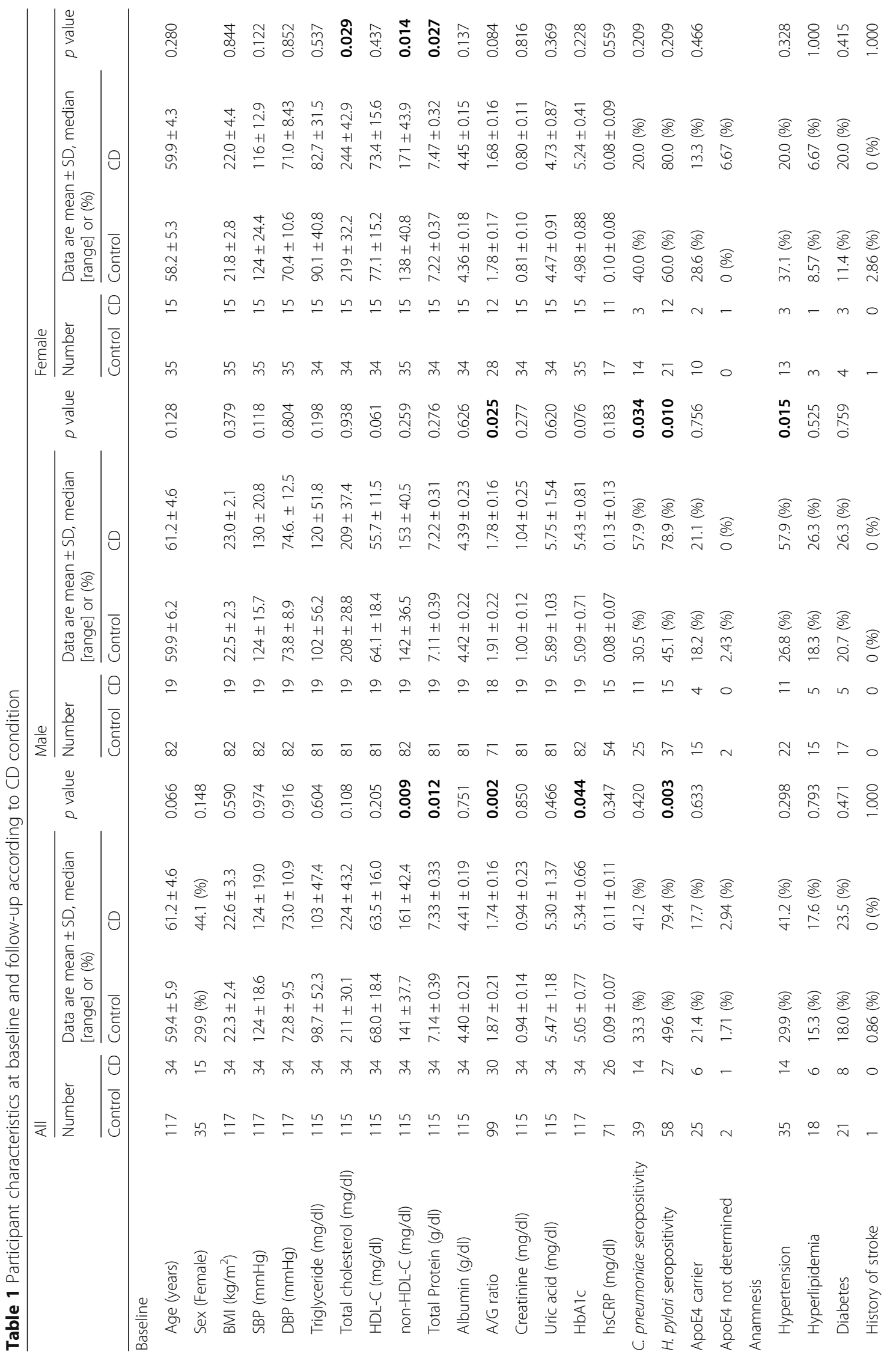




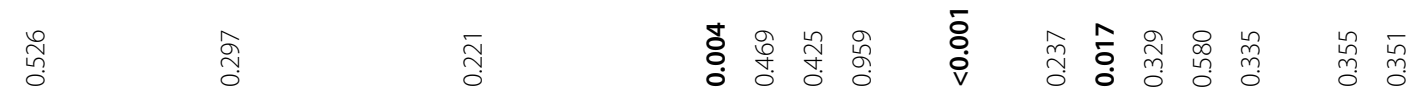

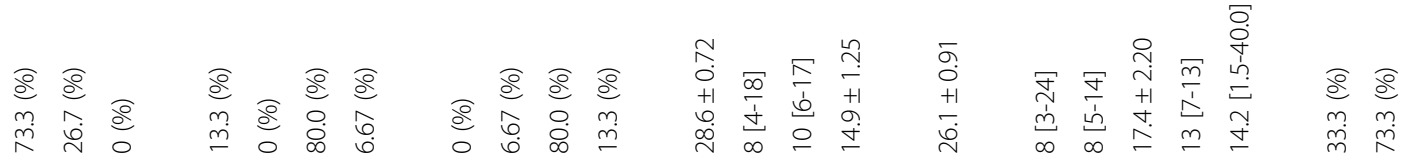

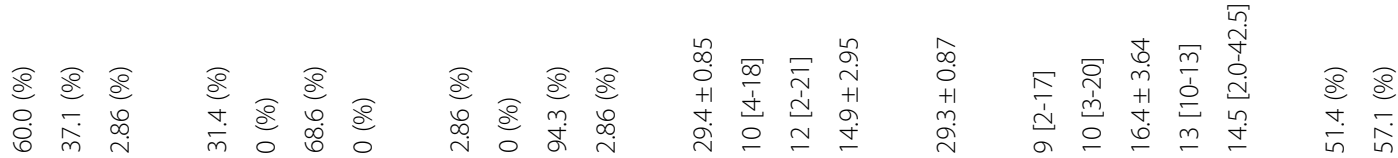

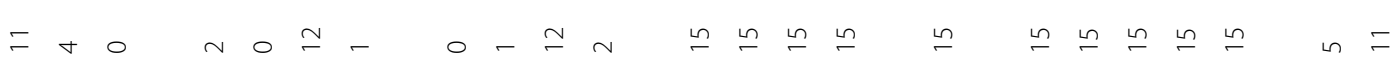

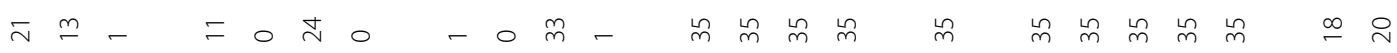

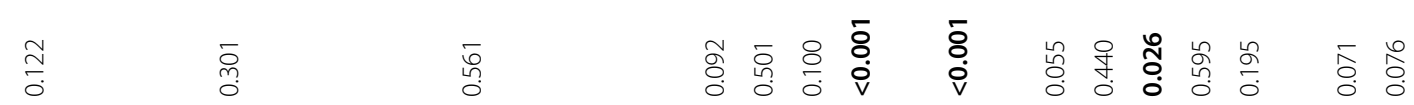

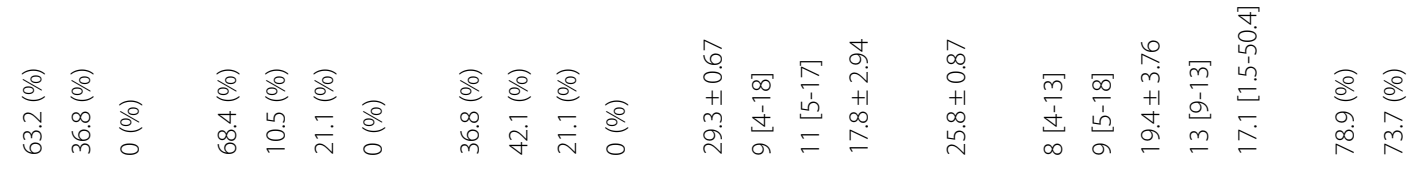

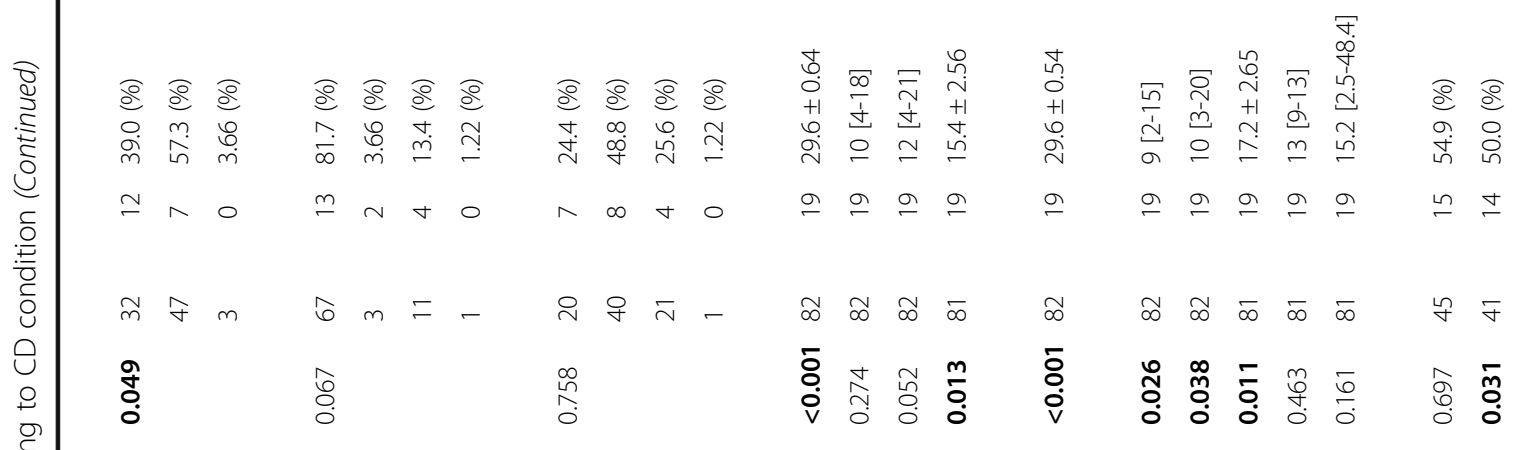

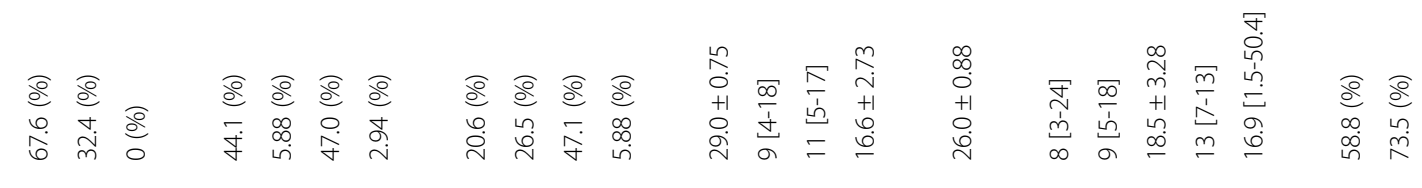

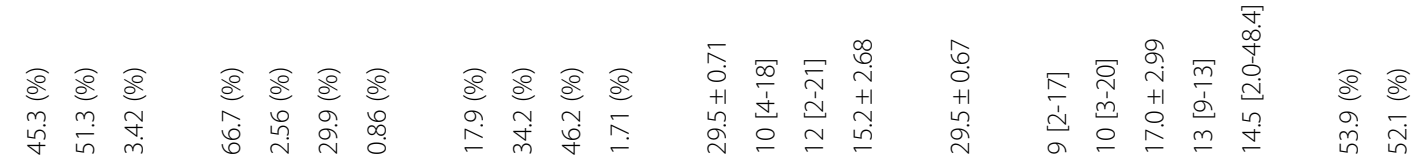

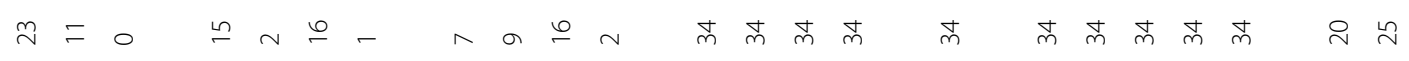

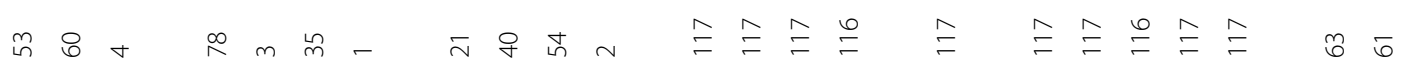

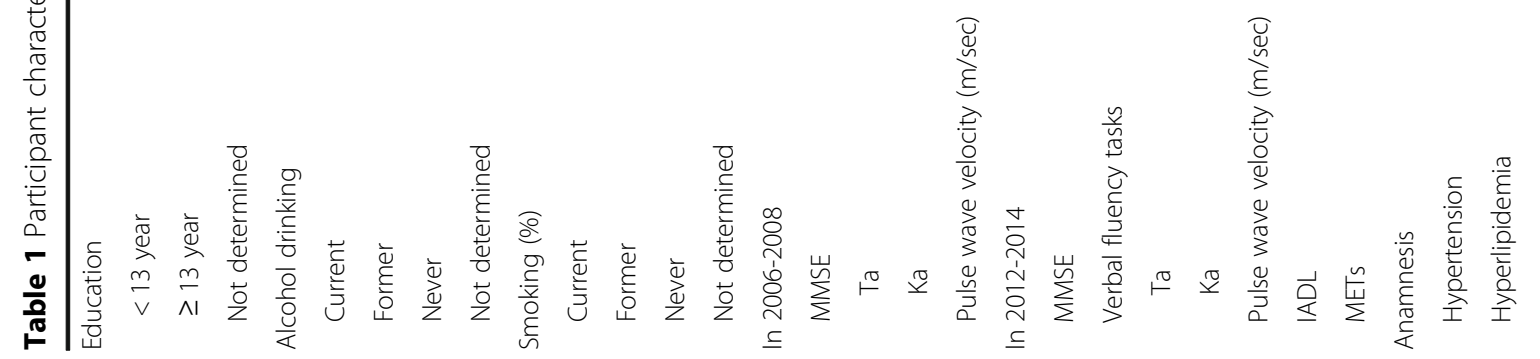




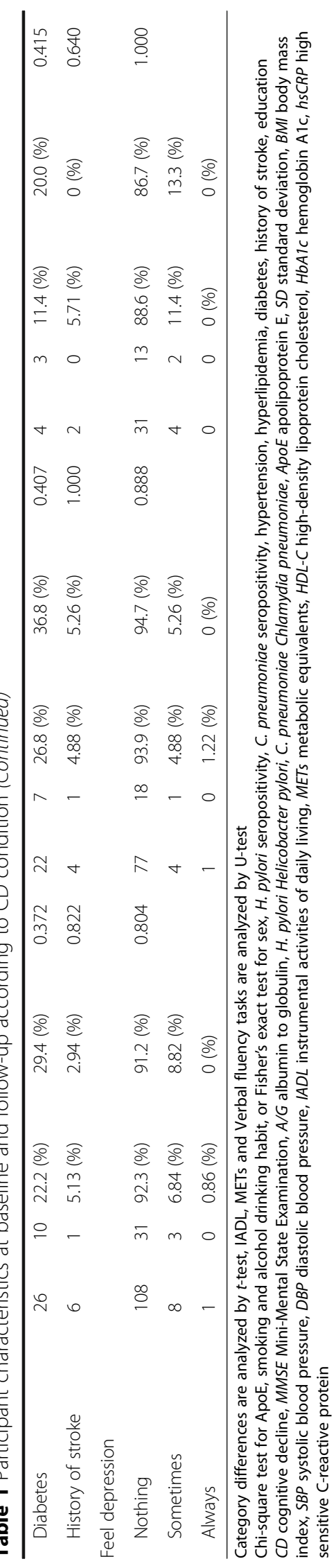


Table 2 Logistic regression analysis according to CD condition

\begin{tabular}{|c|c|c|c|c|c|c|c|c|}
\hline & \multicolumn{2}{|l|}{ Number } & \multicolumn{2}{|l|}{ Model I } & \multicolumn{2}{|l|}{ Model II } & \multicolumn{2}{|l|}{ Model III } \\
\hline & Control & $C D$ & OR & $95 \% \mathrm{Cl}$ & OR & $95 \% \mathrm{Cl}$ & OR & $95 \% \mathrm{Cl}$ \\
\hline \multicolumn{9}{|c|}{ DWL Baseline } \\
\hline grade 0 & 67 & 17 & Reference & & Reference & & Reference & \\
\hline grade 1 & 44 & 17 & 1.476 & $0.669-3.255$ & 1.684 & $0.715-3.967$ & 1.451 & $0.573-3.671$ \\
\hline \multicolumn{9}{|c|}{ DWL 1st follow-up } \\
\hline grade 0 & 52 & 11 & Reference & & Reference & & Reference & \\
\hline grade 1 & 55 & 20 & 1.764 & $0.754-4.129$ & 1.872 & $0.759-4.619$ & 1.763 & $0.697-4.455$ \\
\hline grade 2 & 8 & 3 & 1.314 & $0.620-2.787$ & 1.458 & $0.598-3.557$ & 1.049 & $0.299-3.387$ \\
\hline \multicolumn{9}{|c|}{ DWL 2nd follow-up } \\
\hline grade 0 & 43 & 5 & Reference & & Reference & & Reference & \\
\hline grade 1 & 50 & 21 & 3.659 & $1.242-10.77$ & 4.562 & $1.382-15.05$ & 4.427 & $1.323-14.81$ \\
\hline grade 2 & 17 & 7 & 2.058 & $1.042-4.062$ & 3.969 & $1.424-11.06$ & 4.215 & $1.384-12.83$ \\
\hline grade 3 & 6 & 1 & 1.008 & $0.450-2.259$ & 0.807 & $0.273-2.384$ & 1.103 & $0.274-4.442$ \\
\hline \multicolumn{9}{|c|}{ PVH Baseline } \\
\hline grade 0 & 83 & 23 & Reference & & Reference & & Reference & \\
\hline grade 1 & 29 & 11 & 1.156 & $0.479-2.791$ & 0.848 & $0.327-2.197$ & 0.700 & $0.254-1.930$ \\
\hline \multicolumn{9}{|c|}{ PVH 1st follow-up } \\
\hline grade 0 & 66 & 18 & Reference & & Reference & & Reference & \\
\hline grade 1 & 41 & 15 & 1.152 & $0.498-2.668$ & 0.971 & $0.392-2.409$ & 0.909 & $0.359-2.298$ \\
\hline grade 2 & 8 & 1 & 0.591 & $0.192-1.815$ & 0.611 & $0.188-1.988$ & 0.632 & $0.191-2.095$ \\
\hline \multicolumn{9}{|c|}{ PVH 2st follow-up } \\
\hline grade 0 & 61 & 17 & Reference & & Reference & & Reference & \\
\hline grade 1 & 44 & 13 & 0.857 & $0.354-2.075$ & 0.847 & $0.337-2.131$ & 0.857 & $0.337-2.175$ \\
\hline grade 2 & 7 & 4 & 1.450 & $0.716-2.937$ & 1.254 & $0.554-2.842$ & 1.221 & $0.524-2.841$ \\
\hline
\end{tabular}

Modell: Adjusted for age and sex

Model II: Adjusted for age, sex, apolipoprotein E4, education, smoking and alcohol drinking habits

Model III: Adjusted for age, sex, apolipoprotein E4, education, smoking and alcohol drinking habits, hypertension, hyperlipidemia and diabetes

$C D$ cognitive decline, $D W L$ white matter lesions, $P V H$ perivascular hyperintensities, $O R$ odds ratio, $C l$ confidence interval

dyslipidemia, diabetes, and infectious diseases are plausible candidates. The present results revealed that $H$. pylori seropositivity tended to be related to more severe CD incidence. Furthermore, the present study explored, for the first time, an association between $\mathrm{A} / \mathrm{G}$ ratios and $\mathrm{CD}$ incidence.

Growing evidence has underscored a mechanistic link between cholesterol metabolism in the brain and the formation of amyloid plaques. Excess brain cholesterol has been associated with increased formation and deposition of $\beta$-amyloid from amyloid precursor proteins. Indeed, non-HDL-C was associated with $C D$ incidence in the present study. Cholesterol-lowering statins have become a focus for AD research [24]. Moreover, genetic polymorphisms associated with pivotal points in cholesterol metabolism within brain tissues may contribute to $\mathrm{AD}$ risk and pathogenesis. A recent meta-analysis indicated the positive predictive value of the ApoE4 allele for progression from cognitive impairment to AD-type dementia [25]. Although there is convincing evidence to suggest that
ApoE4 is the main predictor for progression from $C D$ to $\mathrm{AD}, \mathrm{ApoE} 4$ may not be a risk factor for $\mathrm{CD}$ incidence. For instance, the present findings revealed that ApoE4 status was not associated with $\mathrm{CD}$ incidence.

Cognitive impairment can present with mild deficits affecting one or multiple cognitive domains. Size and location of white matter lesions and ischemic and hemorrhagic strokes are associated with varying clinical presentation in these patients [26]. Concerning the link between CD incidence and cerebrovascular lesion occurrence, we found that the CD group showed not only decreased MMSE scores but also progression of DWL Fazekas grade. In general, white matter lesions are a key vascular, cognitive impairment marker. Although DWL and PVH were not predictive of $\mathrm{CD}$ incidence in the present study, $\mathrm{CD}$ group indicated DWL grade progression.

Recent studies have shown that $H$. pylori infection leads to cognitive impairment [3]. H. pylori infection likely influences cognitive impairment by increasing neurodegenerative lesions, especially neurofibrillary tangles 
Table 3 Logistic regression analysis according to CD condition

\begin{tabular}{|c|c|c|c|c|c|c|c|c|}
\hline & \multicolumn{2}{|l|}{ Number } & \multicolumn{2}{|l|}{ Model I } & \multicolumn{2}{|c|}{$\underline{\text { Model II }}$} & \multicolumn{2}{|c|}{ Model III } \\
\hline & Control & $C D$ & OR & $95 \% \mathrm{Cl}$ & OR & $95 \% \mathrm{Cl}$ & $\mathrm{OR}$ & $95 \% \mathrm{Cl}$ \\
\hline BMI & 117 & 34 & 1.073 & $0.998-1.154$ & 1.060 & $0.910-1.235$ & 1.024 & $0.868-1.207$ \\
\hline SBP & 117 & 34 & 0.999 & $0.938-1.151$ & 0.994 & $0.971-1.017$ & 0.971 & $0.941-1.002$ \\
\hline DBP & 117 & 34 & 1.010 & $0.970-1.052$ & 0.998 & $0.954-1.044$ & 0.997 & $0.941-1.036$ \\
\hline Triglyceride & 115 & 34 & 1.003 & $0.996-1.011$ & 1.002 & $0.994-1.010$ & 1.002 & 0.989-1.015 \\
\hline Total cholesterol & 115 & 34 & 1.001 & $0.998-1.022$ & 1.009 & $0.995-1.022$ & 1.009 & $0.995-1.022$ \\
\hline $\mathrm{HDL}-\mathrm{C}$ & 115 & 34 & 0.972 & $0.946-0.999$ & 0.977 & $0.950-1.004$ & 0.973 & $0.942-1.005$ \\
\hline non-HDL-C & 115 & 34 & 1.014 & $1.003-1.025$ & 1.013 & $1.001-1.025$ & 1.013 & $1.001-1.027$ \\
\hline Total Protein & 115 & 34 & 2.971 & $1.023-8.622$ & 3.575 & $1.088-11.74$ & 3.219 & 0.938-11.04 \\
\hline Albumin & 115 & 34 & 2.035 & $0.291-14.21$ & 1.980 & $0.243-16.12$ & 1.852 & $0.218-15.71$ \\
\hline A/G ratio & 99 & 30 & 0.063 & $0.006-0.619$ & 0.032 & $0.003-0.379$ & 0.037 & $0.003-0.470$ \\
\hline Creatinine & 115 & 34 & 2.688 & $0.207-34.86$ & 2.852 & $0.185-43.87$ & 2.235 & $0.135-36.88$ \\
\hline Uric acid & 115 & 34 & 1.007 & 0.695-1.459 & 1.037 & $0.707-1.520$ & 1.068 & $0.730-1.563$ \\
\hline $\mathrm{HbA} 1 \mathrm{c}$ & 117 & 34 & 2.433 & $1.156-5.118$ & 2.405 & $1.131-5.112$ & 2.586 & $1.036-6.455$ \\
\hline hsCRP & 71 & 26 & 12.95 & $0.104-1617$ & 66.97 & $0.303-14824$ & 42.42 & $0.127-14225$ \\
\hline C. pneumoniae seropositivity & 117 & 34 & 1.297 & $0.580-2.899$ & 1.437 & $0.619-3.336$ & 1.593 & $0.664-3.82$ \\
\hline H. pylori seropositive & 117 & 34 & 3.507 & $1.398-8.801$ & 4.867 & $1.754-13.50$ & 4.786 & $1.710-13.39$ \\
\hline Pulse wave velocity in $2006-2008$ & 116 & 34 & 1.184 & $1.021-1.371$ & 1.209 & $1.028-1.422$ & 1.179 & $0.989-1.404$ \\
\hline Pulse wave velocity in 2012-2014 & 116 & 34 & 1.158 & $1.015-1.322$ & 1.145 & $0.989-1.327$ & 1.125 & $0.963-1.313$ \\
\hline Education & 113 & 34 & 2.129 & $0.927-4.890$ & & & & \\
\hline ApoE4 carrier & 115 & 33 & 0.781 & $0.310-1.970$ & & & & \\
\hline
\end{tabular}

Model I: Adjusted for age and sex

Model II: Adjusted for age, sex, apolipoprotein E4, education, smoking and alcohol drinking habits

Model III: Adjusted for age, sex, apolipoprotein E4, education, smoking and alcohol drinking habits, hypertension, hyperlipidemia and diabetes

$C D$ cognitive decline, $O R$ odds ratio, $C l$ confidence interval, $A / G$ albumin to globulin, $H$. pylori Helicobacter pylori, $C$. pneumoniae Chlamydia pneumoniae, ApoE apolipoprotein $\mathrm{E}, B M I$ body mass index, SBP systolic blood pressure, DBP diastolic blood pressure, $H D L-C$ high-density lipoprotein cholesterol, $H b A 1 C$ hemoglobin $\mathrm{A} 1 \mathrm{C}$, hsCRP high sensitive $\mathrm{C}$-reactive protein

and neuronal loss via ischemic lesions. $H$. pylori infection evolving over many years could also cause chronic gastric and plasmatic inflammation, thus inducing a chronic inflammation model plausibly responsible for cerebrovascular lesions and the exacerbation of neurodegeneration [3]. Moreover, when accomplished, $H$. pylori eradication is beneficial for improving cognitive and functional states

Table 4 Multiple logistic regression analysis with stepwise forward selection based on CD condition

\begin{tabular}{llllll}
\hline & \multicolumn{2}{l}{ Multivariate } & & \multicolumn{2}{l}{ Stepwise forward selection } \\
\cline { 2 - 3 } non-HDL-C & OR & $95 \% \mathrm{Cl}$ & & OR & $95 \% \mathrm{Cl}$ \\
\hline A/G ratio & 1.011 & $0.999-1.024$ & & \\
HbA1C & 0.265 & $0.022-3.215$ & $\mathbf{0 . 0 9 2}$ & $\mathbf{0 . 0 1 0 - 0 . 8 8 7}$ \\
H. pylori seropositive & $\mathbf{4 . 2 5 5}$ & $\mathbf{1 . 4 2 2}-\mathbf{1 2 . 7 3}$ & $\mathbf{4 . 4 6 8}$ & $\mathbf{1 . 5 3 5 - 1 3 . 0 0}$ \\
Sex & 1.743 & $0.782-3.883$ & & \\
Age & 1.493 & $0.522-4.270$ & & \\
\hline
\end{tabular}

$C D$ cognitive decline, $O R$ odds ratio, $C l$ confidence interval, $H D L-C$ high-density lipoprotein cholesterol, $H b A 1 c$ hemoglobin A1c, A/G albumin to globulin, H. pylori: Helicobacter pylori among patients, perhaps altering the progressive nature of $\mathrm{AD}$ [27]. Additionally, chronic inflammation might be an underlying factor for an association between metabolic syndrome and $\mathrm{CD}[28]$. The present study suggests a relationship between inflammation, disruption of homeostatic factors [e.g., cholesterol metabolism (dyslipidemia), HbA1c

Table 5 Correlations between $A / G$ ratio and variables used in the multivariate analysis

\begin{tabular}{lll}
\hline & A/G ratio & \\
\cline { 2 - 3 } & Coefficient & $p$ value \\
\hline MMSE score in 2006-2008 & 0.187 & $\mathbf{0 . 0 3 4}$ \\
MMSE score in 2012-2014 & 0.264 & $\mathbf{0 . 0 0 3}$ \\
non-HDL-C & -0.230 & $\mathbf{0 . 0 0 9}$ \\
HbA1C & -0.193 & $\mathbf{0 . 0 2 9}$ \\
hsCRP & -0.369 & $\mathbf{0 . 0 0 1}$ \\
Pulse wave velocity in 2006-2008 & -0.047 & 0.598 \\
Pulse wave velocity in 2012-2014 & -0.001 & 0.989 \\
\hline
\end{tabular}

A/G albumin to globulin, MMSE Mini-Mental State Examination, HDL-C highdensity lipoprotein cholesterol, HbA1c hemoglobin A1c, hsCRP high sensitive C-reactive protein 
(diabetes), and H. pylori seropositivity (infectious disease)] and cognitive function, since these inflammatory mechanisms are also hypothesized to be involved in the pathogenesis of cognitive impairment. Furthermore, inflammation may also promote the development and progression of atherosclerotic plaques [8], which is in line with evidence suggesting a link between cognitive impairment and atherosclerosis [9]. However, in the present study, pulse wave velocity in 2006-2008 was not predictive of $\mathrm{CD}$. In other words, disruption of homeostatic factors, in itself, was a more useful predictor of $C D$ incidence than arterial stiffness.

From a preventive viewpoint, albumin serves as an antioxidant, eliminates toxins, and inhibits the formation of amyloid beta-peptide fibrils. Several studies suggest that low albumin levels are associated with a risk for cognitive impairment and dementia [29,30]. The present study, however, observed that CD incidence was associated with $\mathrm{A} / \mathrm{G}$ ratios but not albumin. In fact, albumin levels did not differ between the control and CD groups. Additionally, total protein levels trended toward a risk for CD incidence, indicating that globulin levels were increased in the $\mathrm{CD}$ group due to no difference in albumin levels between the control and CD groups. Namely, A/G ratios may decrease due to globulin levels rising during chronic inflammation. Similarly, increased serum globulins have been associated with cancer, rheumatoid diseases, chronic liver disease, nephrotic syndrome, and diabetes mellitus; decreased albumin has been associated with chronic infections, chronic liver disease, and nephrotic syndrome [31,32]. Thus, it appears that the modification of albumin and globulin is associated with disruption of homeostasis. In the present study, A/G ratios were also significantly and positively correlated with MMSE scores and negatively correlated with cholesterol metabolism, HbA1c, and hsCRP. These factors were decreased in relation to CD incidence based on our stepwise regression analysis. In sum, the A/G ratio may be a very reliable index for $\mathrm{CD}$ incidence caused by disruption of homeostasis.

A few study limitations should be noted. First, there were a relatively small number of participants in the $C D$ group. Therefore, an analysis of data from male and female participants separately would not be useful because of the low statistical power. Although, the proportion of male and female participants, and the education level of the participants differed between the two groups, logistic regression analysis was performed after adjusting for these variables. While a study with low statistical power has a reduced likelihood of detecting a true effect, nested case-control studies with small sample sizes are still widely conducted and can be used to identify candidate targets. Secondly, we diagnosed $H$. pylori infections via serum antibody detection, whereas the gold standard involves gastric testing. The primary limitation of this serologic test is its inability to discriminate between current and old infections. However, $H$. pylori induces humoral and cellular immune responses that can affect or perpetuate neural tissue damage [33]. This pathogen may influence the pathophysiology of $\mathrm{AD}$ by inducing vascular disorders that have been implicated in endothelial damage and neurodegeneration. Overall, the results of the present and previous studies suggest that both current and old $H$. pylori infections contribute to $\mathrm{CD}$ by inducing neural tissue damage. One other issue was that $\mathrm{A} / \mathrm{G}$ ratios, as well as other biological markers, were only determined once, during the baseline survey. Conversely, cognitive data were available at both baseline and follow-up. Therefore, larger prospective trials are needed to better assess how A/G ratios are associated with CD incidence.

\section{Conclusions}

The current study observed that $\mathrm{A} / \mathrm{G}$ ratios, which are part of routinely administered laboratory tests, could reflect changes in homeostatic factors. Additional investigations are expected to show that the modification of A/ $\mathrm{G}$ ratios could lead toward novel and effective strategies for predictive $\mathrm{CD}$ screening.

\begin{abstract}
Abbreviations
A/G: Albumin to globulin; AD: Alzheimer's disease; ApoE: Apolipoprotein E; BMI: Body mass index; C. pneumoniae: Chlamydia pneumoniae; CD: Cognitive decline; Cl: Confidence interval; DBP: Diastolic blood pressure; DWL: White matter lesions; H. pylori: Helicobacter pylori; HbA1c: Hemoglobin A1c; HDL-C: High-density lipoprotein cholesterol; hsCRP: High sensitive C-reactive protein; IADL: Instrumental activities of daily living; METs: Metabolic equivalents; MMSE: Mini-mental state examination; MRI: Magnetic resonance imaging; OR: Odds ratio; PVH: Periventricular hyperintensities; SBP: Systolic blood pressure; SD: Standard deviation
\end{abstract}

\section{Acknowledgements}

Not applicable.

\section{Funding}

This study was supported in part by Grant-in-Aid for Scientific Research on Priority Areas (No. 17015018), Grant-in-Aid for Scientific Research on Innovative Areas (No. 221S0001) from the Japanese Ministry of Education, Culture, Sports, Science, and Technology. JSPS KAKENHI Grant Number $16 \mathrm{H} 06277,23390176$ and 19390178 supported this work.

\section{Availability of data and materials}

The dataset used in this article is not published, but anonymous data can be available at request to the authors.

\section{Authors' contributions}

TKoyama analyzed the data, and wrote the manuscript. NK, MN, TM, YW designed the idea of the study. EO, DM, IW, FM, MK, TKasai, YO, TY, TT, IM, SM collected the samples. AT, KY, KT were in charge of the MR evaluations. All authors contributed to approval of the manuscript.

Competing interests

The authors declare that they have no competing interests.

Consent for publication

Not applicable. 


\section{Ethics approval and consent to participate}

The Ethics Board from the Kyoto Prefectural University of Medicine approved the study protocol (G-144). After we explained the purpose of the study, written informed consent was obtained from all participants.

\section{Author details}

'Department of Epidemiology for Community Health and Medicine, Kyoto Prefectural University of Medicine, 465 Kajii-cho, Kamigyo-ku, Kyoto 602-8566, Japan. ${ }^{2}$ Department of Dental Medicine, Kyoto Prefectural University of Medicine, 465 Kajii-cho, Kamigyo-ku, Kyoto 602-8566, Japan. ${ }^{3}$ Department of Neurology, Kyoto Prefectural University of Medicine, 465 Kajii-cho, Kamigyo-ku, Kyoto 602-8566, Japan. ${ }^{4}$ Department of Pharmacology, Kyoto Prefectural University of Medicine, 465 Kajii-cho, Kamigyo-ku, Kyoto 602-8566, Japan. ${ }^{5}$ Department of Molecular Pathobiology of Brain Diseases, Kyoto Prefectural University of Medicine, 465 Kajii-cho, Kamigyo-ku, Kyoto 602-8566, Japan. ${ }^{6}$ Endoscopy Department, Kindai University Nara Hospital, 1248-1 Otoda-cho, Ikoma 630-0293, Japan. ${ }^{7}$ Department of Radiology, Kyoto Prefectural University of Medicine, Kyoto, Japan. ${ }^{8}$ Kyoto Industrial Health Association, 67 Nishinokyo Kitatsuboi-cho, Nakagyo-ku, Kyoto 604-8472, Japan. ${ }^{9}$ Director of North Medical Center, Kyoto Prefectural University of Medicine, 481 Aza-Otokoyama, Yosano-cho, Yosa-gun, Kyoto 629-2261, Japan.

Received: 12 August 2016 Accepted: 30 November 2016 Published online: 08 December 2016

\section{References}

1. Ritchie K, Touchon J. Mild cognitive impairment: conceptual basis and current nosological status. Lancet. 2000;355(9199):225-8.

2. DeCarli C. Mild cognitive impairment: prevalence, prognosis, aetiology, and treatment. Lancet Neurol. 2003;2(1):15-21.

3. Roubaud-Baudron C, Krolak-Salmon P, Quadrio I, Megraud F, Salles N. Impact of chronic Helicobacter pylori infection on alzheimer's disease: preliminary results. Neurobiol Aging. 2012;33(5):1009. e11-1009.

4. Katan M, Moon YP, Paik MC, Sacco RL, Wright CB, Elkind MS. Infectious burden and cognitive function: the Northern Manhattan study. Neurology. 2013:80(13):1209-15

5. Wozniak MA, Itzhaki RF. Antiviral agents in alzheimer's disease: hope for the future? Ther Adv Neurol Disord. 2010;3(3):141-52.

6. Maher PA, Schubert DR. Metabolic links between diabetes and alzheimer's disease. Expert Rev Neurother. 2009;9(5):617-30.

7. Gorelick PB. Role of inflammation in cognitive impairment: results of observational epidemiological studies and clinical trials. Ann N Y Acad Sci. 2010;1207:155-62

8. Libby P, Ridker PM, Maseri A. Inflammation and atherosclerosis. Circulation 2002;105(9):1135-43

9. Iadecola C. The pathobiology of vascular dementia. Neuron. 2013;80(4):844-66.

10. Etgen T, Sander D, Bickel H, Forstl H. Mild cognitive impairment and dementia: the importance of modifiable risk factors. Dtsch Arztebl Int. 2011;108(44):743-50.

11. Ohshima Y, Mizuno T, Yamada K, Matsumoto S, Nagakane Y, Kondo M, Kuriyama N, Miyazaki T, Takeda K, Nishimura T, et al. Low vitamin and carotenoid levels are related to cerebral white matter lesions. J Nutr Health Aging. 2013;17(5):456-60.

12. Folstein MF, Folstein SE, McHugh PR. "Mini-mental state". A practical method for grading the cognitive state of patients for the clinician. J Psychiatr Res. 1975;12(3):189-98.

13. Kuriyama N, Mizuno T, Ohshima Y, Yamada K, Ozaki E, Shigeta M, Mitani S, Kondo M, Matsumoto S, Takeda K, et al. Intracranial deep white matter lesions (DWLs) are associated with chronic kidney disease (CKD) and cognitive impairment: a 5-year follow-up magnetic resonance imaging (MRI) study. Arch Gerontol Geriatr. 2013;56(1):55-60.

14. O'Bryant SE, Humphreys JD, Smith GE, Ivnik RJ, Graff-Radford NR, Petersen RC, Lucas JA. Detecting dementia with the mini-mental state examination in highly educated individuals. Arch Neurol. 2008;65(7):963-7.

15. Henry JD, Crawford JR, Phillips LH. Verbal fluency performance in dementia of the alzheimer's type: a meta-analysis. Neuropsychologia. 2004:42(9):1212-22.

16. Tomioka K, Kurumatani N, Hosoi H. Social participation and the prevention of decline in effectance among community-dwelling elderly: a population-based cohort study. PLoS One. 2015;10(9):e0139065.
17. Koyama T, Matsui D, Kuriyama N, Ozaki E, Tanaka K, Oze I, Hamajima N, Wakai K, Okada R, Arisawa K, et al. Genetic variants of SLC17A1 are associated with cholesterol homeostasis and hyperhomocysteinaemia in Japanese men. Sci Rep. 2015:5:15888.

18. Nasreddine ZS, Phillips NA, Bedirian V, Charbonneau S, Whitehead V, Collin I, Cummings $\mathrm{J}$, Chertkow $\mathrm{H}$. The Montreal cognitive assessment, MoCA: a brief screening tool for mild cognitive impairment. J Am Geriatr Soc. 2005;53(4):695-9.

19. Yamamoto $H$, Watanabe $T$, Miyazaki A, Katagiri $T$, Idei T, Iguchi T, Mimura M, Kamijima K. High prevalence of Chlamydia pneumoniae antibodies and increased high-sensitive C-reactive protein in patients with vascular dementia. J Am Geriatr Soc. 2005;53(4):583-9.

20. Obata Y, Kikuchi S, Miwa H, Yagyu K, Lin Y, Ogihara A. Diagnostic accuracy of serological kits for Helicobacter pylori infection with the same assay system but different antigens in a Japanese patient population. J Med Microbiol. 2003;52(Pt 10):889-92.

21. Munakata M. Brachial-ankle pulse wave velocity: background, method, and clinical evidence. Pulse (Basel, Switzerland). 2016;3(3-4):195-204.

22. Wenham PR, Price WH, Blandell G. Apolipoprotein E genotyping by one-stage PCR. Lancet. 1991:337(8750):1158-9.

23. Fazekas F, Chawluk JB, Alavi A, Hurtig HI, Zimmerman RA. MR signal abnormalities at $1.5 \mathrm{~T}$ in alzheimer's dementia and normal aging. AJR Am J Roentgenol. 1987;149(2):351-6.

24. Shobab LA, Hsiung GY, Feldman HH. Cholesterol in alzheimer's disease. Lancet Neurol. 2005;4(12):841-52.

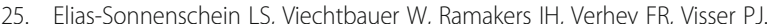
Predictive value of APOE-epsilon4 allele for progression from $\mathrm{MCI}$ to AD-type dementia: a meta-analysis. J Neurol Neurosurg Psychiatry. 2011;82(10):1149-56.

26. Farooq MU, Gorelick PB. Vascular cognitive impairment. Curr Atheroscler Rep. 2013;15(6):330

27. Kountouras J, Boziki M, Gavalas E, Zavos C, Grigoriadis N, Deretzi G, Tzilves D, Katsinelos P, Tsolaki M, Chatzopoulos D, et al. Eradication of Helicobacter pylori may be beneficial in the management of alzheimer's disease. J Neurol. 2009;256(5):758-67.

28. Yaffe K, Kanaya A, Lindquist K, Simonsick EM, Harris T, Shorr Rl, Tylavsky FA, Newman AB. The metabolic syndrome, inflammation, and risk of cognitive decline. JAMA. 2004;292(18):2237-42.

29. Kim TS, Pae CU, Yoon SJ, Jang WY, Lee NJ, Kim JJ, Lee SJ, Lee C, Paik IH, Lee CU. Decreased plasma antioxidants in patients with alzheimer's disease. Int $J$ Geriatr Psychiatry. 2006;21(4):344-8.

30. Llewellyn DJ, Langa KM, Friedland RP, Lang IA. Serum albumin concentration and cognitive impairment. Curr Alzheimer Res. 2010;7(1):91-6.

31. Chojkier M. Inhibition of albumin synthesis in chronic diseases: molecular mechanisms. J Clin Gastroenterol. 2005;39(4 Suppl 2):S143-6.

32. Suh B, Park S, Shin DW, Yun JM, Keam B, Yang HK, Ahn E, Lee H, Park JH, Cho B. Low albumin-to-globulin ratio associated with cancer incidence and mortality in generally healthy adults. Ann Oncol. 2014;25(11):2260-6.

33. Kountouras J, Gavalas E, Boziki M, Zavos C. Helicobacter pylori may be involved in cognitive impairment and dementia development through induction of atrophic gastritis, vitamin B-12 folate deficiency, and hyperhomocysteinemia sequence. Am J Clin Nutr. 2007;86(3):805-6. author reply $806-7$.

\section{Submit your next manuscript to BioMed Central and we will help you at every step:}

- We accept pre-submission inquiries

- Our selector tool helps you to find the most relevant journal

- We provide round the clock customer support

- Convenient online submission

- Thorough peer review

- Inclusion in PubMed and all major indexing services

- Maximum visibility for your research

Submit your manuscript at www.biomedcentral.com/submit
C) BioMed Central 